\title{
Interleukin-1 $\beta$ Tear Concentration in Glaucomatous and Ocular Hypertensive Patients Treated With Preservative-Free Nonselective Beta-Blockers
}

\author{
GIANLUCA MANNI, MD, MARCO CENTOFANTI, MD, PHD, FRANCESCO ODDONE, MD, \\ MARIACRISTINA PARRAVANO, MD, AND MASSIMO G. BUCCI, MD
}

- PURPOSE: To evaluate the ocular surface inflammatory response to the presence of preservatives in nonselective beta-blocker eyedrops.

- DESIGN: Prospective, crossover, single-masked, randomized clinical study.

- METHODS: STUDY POPULATION: Twenty primary openangle glaucoma or ocular hypertensive patients were divided in two groups, one treated with preservative-free timolol $0.5 \%$ (group 1) and the other with preserved timolol $0.5 \%$ (group 2) eyedrops. After 60 days of therapy and 3 more weeks of washout, the two groups switched to the other therapy. PROCEDURE: At each visit, basal tear samples were collected from the inferior conjunctival fornix for the determination of interleukin (IL)-1 $\beta$ tear concentrations by an enzyme-linked immunosorbent assay. Intraocular pressure measurement, conjunctival hyperemia, superficial punctate keratitis, and tear film breakup time were evaluated. MAIN OUTCOME MEASURE: IL-1 $\beta$ concentration in tears following the use of preserved eyedrops.

- RESULTS: IL-1 $\beta$ tear concentrations increased significantly in both groups, compared with baseline values, during preserved timolol therapy. There were no statistically significant changes in hyperemia and superficial punctate keratitis throughout the study in either group. A statistically significant breakup time reduction was observed in both groups after 30 days and after 60 days of preserved therapy.

- CONCLUSION: The use of preservatives in timolol $0.5 \%$ eyedrops leads to tear film instability and ocular surface inflammatory changes documented by a reduction of breakup time and an increase of IL-1 $\beta$ tear concen-

\section{Accepted for publication August 16, 2004.}

From the University of Rome "Tor Vergata", Dipartimento di Biopatologie e Diagnostica per Immagini; Fondazione G.B. Bietti for the Study and Research in Ophthalmology, Rome, Italy.

Inquiries to Francesco Oddone, MD, Fondazione G.B. Bietti, Viale Oxford 81, Rome 00133, Italy; e-mail: f.oddone@libero.it trations. Preservative-free beta-blockers are preferable for long-term hypotensive therapy to prevent ocular surface inflammation. (Am J Ophthalmol 2005;139: 72-77. (C) 2005 by Elsevier Inc. All rights reserved.)

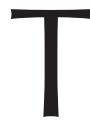

HE IMPORTANCE OF INTRAOCULAR PRESSURE (IOP) in the development of glaucomatous optic neuropathy has recently been confirmed, and a new definition of glaucoma as an "intraocular pressure-sensitive optic neuropathy" has been proposed..$^{1}$ Indeed, it has been demonstrated that lowering IOP with a medical or surgical approach reduces the risk of visual field defect progression in patients with initial or advanced primary open-angle glaucoma and normal tension glaucoma and the development of a glaucomatous defect in patients with ocular hypertension..$^{2-5}$

For medical treatment to be effective, adverse events and side effects must be minimized to promote patient compliance and to allow the continuation of therapy. Beta-blockers were introduced more than 20 years ago and remain among the most widely used hypotensive agents for glaucoma treatment. Their efficacy and safety have been widely investigated. ${ }^{6}$

Studies have shown that preservative-free nonselective and selective beta-blockers are less toxic to the ocular surface than preserved versions, suggesting that topical side effects may be largely a result of the presence of preservatives. ${ }^{7-10}$ The most commonly used preservative in ophthalmic preparations is benzalkonium chloride (BAC), a quaternary ammonium with detergent properties used to limit bacterial contamination in multidose containers, with noxious effects toward the ocular surface and tear film. ${ }^{11}$ Benzalkonium chloride exerts its damaging action mainly through a direct cytotoxic mechanism, accentuated by the cumulative effect of repeated administrations of preserved eyedrops. ${ }^{11}$ In 1994, Baudouin and associates ${ }^{12}$ reported the abnormal expression of inflammatory markers 
such as CD23 and HLA-DR in the absence of clinically evident inflammation in the conjunctiva of patients after repetitive contact with various antiglaucoma eyedrops and their common preservative, BAC. ${ }^{12}$

In recent years, it has been demonstrated that inflammatory cytokines such as interleukin (IL)-1 ( $\alpha$ and $\beta$ ), IL-6, and IL-8, which regulate the activation, differentiation, and proliferation of lymphocytes, monocytes, and other immunocompetent cells, may play a key role in the regulation of ocular surface inflammation. ${ }^{13}$ Corneal concentrations of these cytokines are higher in eyes with distinct inflammatory signs (for example, herpetic keratitis, alkali burns). ${ }^{14}$ In addition, several clinical studies report a significant reduction of inflammation in rheumatoid arthritis and Behçet's disease after treatment with anti-tumor necrosis factor $\alpha(\mathrm{TNF} \alpha)$ and anti-IL-1 $\beta .{ }^{15-17}$ In particular, IL-1 participates in the first steps of the inflammatory reaction and is responsible for the induction of the expression of other cytokines such as IL-6 and IL-8.

The aim of this study was to evaluate IL-1 $\beta$ tear levels and clinical ocular signs in patients treated with preserved versus preservative-free nonselective beta-blockers.

\section{METHODS}

A PROSPECTIVE, CROSSOVER, SINGLE-MASKED, RANDOMized clinical study was conducted on 20 primary openangle glaucoma or ocular hypertension patients treated with timolol maleate $0.5 \%$ in both eyes (nine men, 11 women, mean age $53.15 \pm 12.9$ years). The protocol, approved by the university internal ethics committee, was carried out in agreement with the Declaration of Helsinki (1996), and eligible patients were enrolled in the study during a screening visit after signing an informed consent.

Inclusion criteria were as follows: age 18 years or older, diagnosis of open-angle glaucoma (including pseudoexfoliative, pigmentary, and normal tension glaucoma) or ocular hypertension treated with preserved timolol $0.5 \%$ topical therapy in both eyes, and best-corrected visual acuity of $20 / 80$ or better in both eyes. Exclusion criteria included the need for more than one drug to control IOP, presence of an absolute visual field defect in the central 10 degrees, planned alterations of ongoing systemic therapy that could affect IOP, known allergy or hypersensitivity to the drugs studied or their components, corneal abnormalities that could affect applanation tonometry, filtering surgery or any other kind of ocular surgery performed in the preceding 6 months, uncontrolled systemic diseases, and pregnancy, nursing, or planning a pregnancy.

The diagnosis of glaucoma required at least two reliable Humphrey 24-2 SITA-standard visual field tests performed on different days, classified according to the Glaucoma Hemifield Test (GHT) as "outside normal limits" and affecting the same GHT sector. The diagnosis of ocular hypertension required IOP between 22 and $35 \mathrm{~mm}$
$\mathrm{Hg}$ measured on at least two occasions in one eye and Humphrey 24 to 2 SITA-standard visual field tests classified as "within normal limits" according to the GHT. During the screening visit, visual acuity, biomicroscopy with the evaluation of clinical ocular signs, visual field examination, IOP measurement, and ophthalmoscopy were performed. Intraocular pressure was measured twice in each eye at $9 \mathrm{AM} \pm 1$ hour during each visit, using Goldmann's applanation tonometry, and if the difference between the first and second reading was greater than 2 $\mathrm{mm} \mathrm{Hg}$, a third reading was taken. Intraocular pressure was reported as either the average of two or the median of three readings.

If during the screening visit patients met all the enrollment criteria, topical hypotensive therapy was discontinued for washout purposes. At the baseline visit, after 3 weeks of washout from the previous therapy, patients were randomly divided in two groups (1:1 randomization from a computer-generated randomization list): group 1 began therapy with preservative-free timolol 0.5\% (Timolabak, Théa, Paris, France) and group 2 with preserved timolol 0.5\% eyedrops (Timoptol, MSD Chibret, Paris, France). After 60 days of therapy and 3 more weeks of washout, group 1 switched to preserved timolol, and group 2 switched to preservative-free timolol.

Visits were performed at baseline, 30 and 60 days after the onset of the first therapy, after the second washout, and 30 and 60 days after the onset of the second therapy. Visual acuity, biomicroscopy, and IOP measurements were performed at each visit. Conjunctival hyperemia, superficial punctuate keratitis, and tear film breakup time were also evaluated. Conjunctival hyperemia was assessed at the slit-lamp microscope with low magnification and a broad beam of white light using a standard hyperemia photographic chart as reference. The absence of hyperemia was defined as "no visible vessel dilation," and the presence of hyperemia was defined as "noticeable regional or diffuse vessel dilation." Fluorescein tear breakup time was used as a measure of tear film stability. The time required for the breakup of precorneal tear film after blinking was recorded during each visit before fluorescein staining evaluation.

Superficial punctuate keratitis was chosen as a marker of epithelial surface damage and evaluated by fluorescein-dye staining of the corneal surface. After instillation of one drop of $2 \%$ fluorescein, each eye was examined with slit-lamp microscope using high magnification and a bright, broad beam of cobalt blue light. The same slit-lamp and settings were used throughout the study. Fluorescein staining was graded as present or absent over the entire corneal surface, slightly raising the subject's upper eyelid. The presence of corneal staining was defined as more than one dot of fluorescein staining over the cornel surface. Grading schemes for corneal staining commonly used in dry-eye trials ${ }^{18}$ were not considered because the expected range of staining severity was narrow in this study. To minimize the variability of each evaluation, the same 


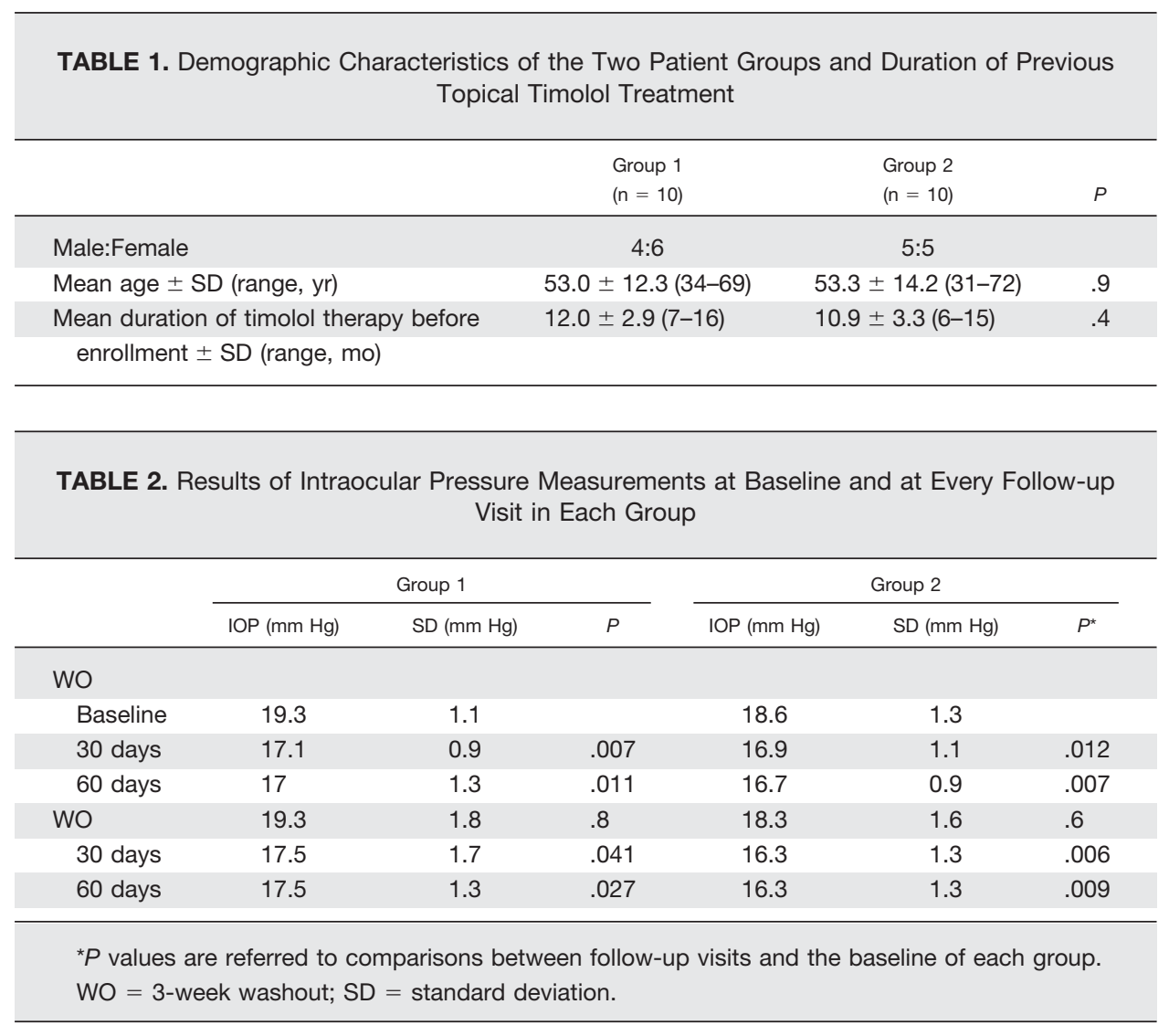

observer performed breakup time, fluorescein staining, and conjunctival hyperemia assessments in a masked fashion throughout the study.

During each visit topical anesthesia was induced by one drop of oxibuprocaine-chloridrate $4 \mathrm{mg}$, to collect at least $20 \mu \mathrm{l}$ of tears from the inferior conjunctival fornix by disposable microcapillaries (Accu-Fill 90 Micropet, Becton-Dickinson, Franklin Lakes, New Jersey). Care was taken to avoid touching the lid margin, corneal surface, and conjunctiva and to avoid trauma. Tears were collected at least 5 minutes after anesthetic instillation to guarantee the anesthetic effect but also to allow absorption of the eyedrop to minimize the amount of oxibuprocaine in the samples. To obtain at least $20 \mu \mathrm{l}$ of tear samples, the collection required several minutes. Patients were informed of the procedure duration and told to blink several times to create new tear film for the collection procedure. The samples collected were immediately frozen at $-80 \mathrm{C}$ and used for the subsequent determination of IL-1 $\beta$ tear concentrations. Cytokine levels in tears were determined by an enzyme-linked immunosorbent assay (ELISA) for IL-1 (R\&D Systems, Minneapolis, Minnesota). All evaluations were performed by a masked investigator.

If both eyes were eligible, the right eye was arbitrarily chosen for the statistical analysis. Paired and unpaired sample $t$ tests were used to compare continuous normal variables for within- and between-group changes. Wilcoxon's signed rank test was used to compare continuous nonnormally distributed paired variables. Fisher's exact test was used to compare the incidence of clinical ocular signs between the two groups. A $P$ value $<.05$ was considered statistically significant.

\section{RESULTS}

ALL 20 PATIENTS COMPLETED THE STUDY AND WERE INcluded in the statistical analysis (group 1: four men and six women; group 2: five men and five women). The mean age of the two groups was statistically similar (group 1:53 \pm 12.3 years, range 34 to 69 years; group $2: 53.3 \pm 14.2$ years, range 31 to 72 years; $P=.9$, Table 1 ). The mean period of treatment with preserved topical beta-blockers before study onset was $12.0 \pm 2.9$ months (range 7 to 16 ) and $10.9 \pm 3.3$ (6 to 15 ) months, respectively, for groups 1 and $2(P=.4)$.

Mean baseline IOP was $19.3 \pm 1.1 \mathrm{~mm} \mathrm{Hg}$ in group 1 and $18.6 \pm 1.3 \mathrm{~mm} \mathrm{Hg}$ in group 2 (nonsignificant). Both treatments were equally effective in reducing IOP throughout the study (Table 2). IL-1 $\beta$ tear levels at baseline were $49.9 \pm 2 \mathrm{pg} / \mathrm{ml}$ and $32.4 \pm 10 \mathrm{pg} / \mathrm{ml}$ in groups 1 and 2 , respectively $(P=.001)$. IL-1 $\beta$ tear concentrations were significantly higher in both groups after both 30 days and 60 days of preserved timolol therapy compared with baseline values. During preservative-free therapy, IL-1 $\beta$ concentrations did not show significant changes compared 
TABLE 3. IL-1 $\beta$ Tear Levels, Expressed in pg/ml

\begin{tabular}{|c|c|c|c|c|c|c|c|c|}
\hline & wo & Baseline & 30 Days & 60 Days & wo & т0а & 30 Days & 60 Days \\
\hline Group 1 & - & $49.9 \pm 20$ & $\begin{array}{l}46.9 \pm 5.3 \\
(P=.138)\end{array}$ & $\begin{array}{l}57.1 \pm 7.8 \\
(P=.063)\end{array}$ & - & $\begin{array}{l}51.6 \pm 7.4 \\
(P=.46)\end{array}$ & $\begin{array}{l}59.8 \pm 6.7 \\
(P=.018)\end{array}$ & $\begin{array}{l}95.5 \pm 5.4 \\
(P=.043)\end{array}$ \\
\hline Group 2 & - & $32.4 \pm 10$ & $\begin{array}{l}53.2 \pm 5.8 \\
(P=.018)\end{array}$ & $\begin{array}{l}88.5 \pm 9.8 \\
(P=.012)\end{array}$ & - & $\begin{array}{l}36.3 \pm 8.9 \\
(P=.9)\end{array}$ & $\begin{array}{l}43.4 \pm 8.8 \\
(P=.14)\end{array}$ & $\begin{array}{l}46.1 \pm 7.3 \\
(P=.067)\end{array}$ \\
\hline
\end{tabular}

WO = 3-week washout; TOa $=$ baseline after the second washout.

In grey are highlighted cells containing IL-1 $\beta$ values during preserved timolol treatment in each group. $P$ values refer to the mean difference between each follow-up visit and its baseline and between the two baselines within each group.

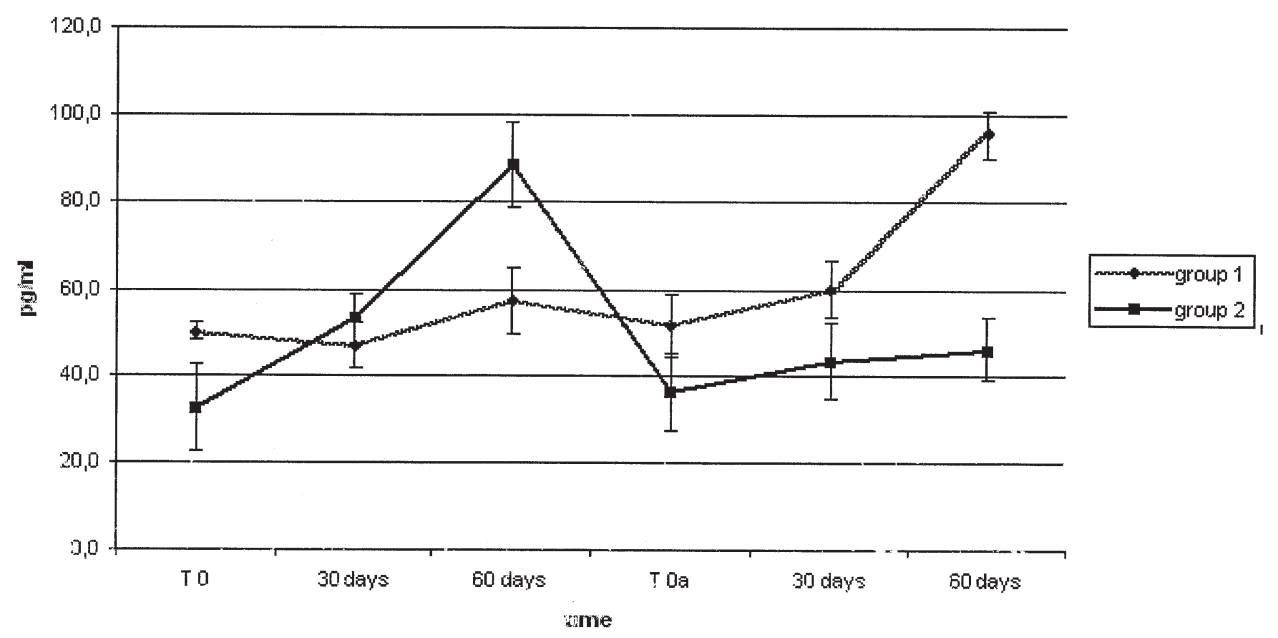

FIGURE 1. Interleukin (IL)-1 $\beta$ tear concentration: mean values and standard deviations. The IL-1 $\beta$ concentration trend was similar in both groups, rising significantly during preserved therapy and decreasing after washout. A slight increase was also observed in both groups during preservative-free therapy, although this increase was not significant.

with baseline values in either group at either time point (Table 3, Figure 1).

There were no statistically significant changes in hyperemia and superficial punctate keratitis in either group at baseline and after each therapy. In both groups, there was a statistically significant breakup time reduction both after 30 days and after 60 days of preserved therapy (Table 4).

\section{DISCUSSION}

IN THIS CROSSOVER STUDY, WE INVESTIGATED THE BEHAVior of IL-1 $\beta$ tear levels during preserved and preservativefree timolol topical therapy. To our knowledge, the increase of IL-1 $\beta$ tear concentrations in human subjects treated with preserved topical beta-blockers has not yet been reported.

Our results are in agreement with a recent comparative investigation by Pisella and associates, ${ }^{19}$ who evaluated, with a different technique, the inflammatory status of the ocular surface after treatment with preserved and preservative-free antiglaucoma drugs. These authors demonstrated that timolol, when preserved with BAC, induces significantly higher levels of inflammation in the conjunctival epithelium than its preservative-free counterpart.

We chose to evaluate IL-1 $\beta$ levels because it is produced by corneal and limbal epithelial cells, and its presence has been demonstrated in tears in basal conditions. ${ }^{24}$ IL-1 ( $\alpha$ and $\beta$ ) is a member of a network of inflammatory cytokines that play a key role in the regulation of ocular surface inflammation, participating, with $\mathrm{TNF}-\alpha$, in the first stages of inflammatory reactions. ${ }^{13} \mathrm{IL}-1$ is a potent inductor of other inflammatory mediators such as IL-6, IL-8, TNF- $\alpha$, and granulocyte macrophage colony stimulating factor (GM-CSF) in ocular tissues. It also stimulates the production of metalloproteinase enzymes in corneal stromal cells, induces the centripetal movement of Langerhans' cells, and may act as mediator of keratocyte apoptosis. ${ }^{20-22}$ The inflammatory activity of IL- 1 is balanced by IL- 1 receptor antagonist, as has been investigated in other tissues. ${ }^{23}$

Previous reports have shown that basal IL-1 $\beta$ tear concentrations in normal subjects ranged from $12.8 \pm 2.3$ $\mathrm{pg} / \mathrm{ml}$ to $29.8 \pm 10 \mathrm{pg} / \mathrm{ml},{ }^{25}$ and thus significantly lower values than those found at the beginning of our study, after 3 weeks of washout, when levels ranged from $32.3 \pm 9.9$ $\mathrm{pg} / \mathrm{ml}$ to $51.6 \pm 7.3 \mathrm{pg} / \mathrm{ml}$. 
TABLE 4. Fluorescein Tear Breakup Time (BT) Expressed in Seconds

\begin{tabular}{ccccccccc}
\hline & WO & Baseline & 30 Days & 60 Days & Wo & TOa & 30 Days & 60 Days \\
\hline Group 1 & - & $8.8 \pm 1.3$ & $8.5 \pm 1.5$ & $9.0 \pm 1.1$ & - & $9.0 \pm 1.1$ & $7.2 \pm 0.9$ & $7.2 \pm 0.8$ \\
& & & $(P=.67)$ & $(P=.76)$ & & $\begin{array}{c}(P=.7) \\
(P=.016)\end{array}$ & $(P=.017)$ \\
Group 2 & - & $8.9 \pm 1.1$ & $7.5 \pm 2.0$ & $7.6 \pm 1.6$ & - & $9.2 \pm 0.9$ & $9.0 \pm 1.1$ & $8.9 \pm 1.4$ \\
& & & $(P=.042)$ & $(P=.041)$ & $(P=.7)$ & $(P=.7)$ & $(P=.4)$ \\
\hline
\end{tabular}

WO = 3-week washout; TOa $=$ baseline after the second washout.

$P$ values refer to the mean difference between each follow-up visit and its baseline and between the two baselines within each group. In grey are highlighted cells containing IL-1 $\beta$ values during preserved timolol treatment in each group.

This difference could be explained considering that our study population did not include normal subjects, and IL-1 $\beta$ tear concentrations might be higher in patients undergoing long-term treatment with topical preserved eyedrops, even after 3 weeks of washout. It should also be noted that after the second washout, IL-1 $\beta$ tear levels decreased to baseline values, and an improvement of breakup time was observed. These findings suggest that, in the short-term, the interruption of preserved eyedrops is beneficial to the ocular surface, but a longer period of suspension may be required to normalize IL-1 $\beta$ tear levels.

IL-1 $\beta$ tear levels showed a statistically significant difference between the two groups at baseline, with higher IL- $1 \beta$ values in group 1 . Because the aim of this study was to evaluate the trend in IL-1 $\beta$ tear levels during preserved and unpreserved topical therapy within each group, a significant difference between groups at baseline does not invalidate the results. It is unlikely that this difference is related to measurement variability, because the ELISA test is highly sensitive. In addition, the strength of the crossover design allowed the confirmation of the same trend in both groups.

Values of IL-1 $\beta$ showed a slow increase from baseline even under unpreserved timolol therapy in both groups. Although the increase was not statistically significant, it may suggest a possible inflammatory activation during unpreserved therapy, although certainly slower and weaker than that induced by preserved therapy. On the clinical side, however, tear film stability measured by means of fluorescein tear breakup time remained unchanged during unpreserved therapy but worsened during preserved therapy, suggesting that the lack of preservatives and the consequent lower inflammatory status have an important clinical impact. The results of a recent study by Pisella and associates ${ }^{19}$ confirm that a low inflammatory status is maintained up to 1 year during treatment with preservative-free timolol. ${ }^{19}$

It has been reported that topically applied BAC decreases the density of goblet cells ${ }^{19}$ and increases the dissolution of the conjunctival mucin layer adsorbed on the surface of the corneal epithelium. ${ }^{26}$ This supports the finding of tear film instability, documented by a reduction of breakup time, observed in our study after treatment with preserved beta-blockers. These alterations may lead to subtle signs of chronic ocular toxicity, such as superficial punctate keratitis, with long-term consequences.

In our study the incidence of hyperemia and superficial punctuate keratitis was low after the washout periods, with no significant changes throughout the study in either group. This suggests that 2 months of preserved topical monotherapy are not sufficient to develop signs of epithelial trauma, which is commonly observed during long-term glaucoma therapies, especially those therapies using of more than one preserved eyedrop. However, the presence of elevated IL-1 $\beta$ tear concentrations and the reduction of breakup time suggest an inflammatory activation that may, in time, lead to clinical ocular surface alterations.

Histopathologic and impression cytology studies have demonstrated inflammation, squamous metaplasia, and subconjunctival fibrosis in the conjunctiva and Tenon's capsule of patients chronically treated with preserved eyedrops ${ }^{8}$ and the failure of filtering surgery for glaucoma is reported to occur more frequently in patients receiving long-term topical treatment.27,28 Therefore, the use of preserved eyedrops should be avoided in candidates for filtration surgery to prevent the induction of any ocular surface inflammation, even if subclinical, that may increase the risk of bleb failure.

The results of our study show that the tear film alterations observed during the administration of topical preserved therapies are likely related to the inflammatory response of the ocular surface. The mechanisms of this inflammatory response involve an increase of IL-1 $\beta$. Thus, the use of preservative-free beta-blockers should be preferred for the treatment of chronic glaucoma to avoid topical side effects and improve patient adherence to therapy.

\section{REFERENCES}

1. Lichter PR. Expectations from clinical trials. Results of the Early Manifest Glaucoma Trial. Arch Ophthalmol 2002;120: 1371-1372.

2. Gordon MO, Beiser JA, Brandt JD, et al. The Ocular Hypertension Treatment Study: a randomized trial deter- 
mines that topical ocular hypotensive medication delays or prevents the onset of primary open-angle glaucoma. Arch Ophthalmol 2002;120:701-713.

3. Heijl A, Leske MC, Bengtsson B, et al, for the Early Manifest Glaucoma Trial Group. Reduction of intraocular pressure and glaucoma progression: results from the Early Manifest Glaucoma Trial. Arch Ophthalmol 2002;120:1268-1279.

4. Collaborative Normal Tension Glaucoma Study Group. Comparison of glaucomatous progression between untreated patients with normal-tension glaucoma and patients with therapeutically reduced intraocular pressures. Am J Ophthalmol 1998;126:487-497.

5. The AGIS Investigators. The Advanced Glaucoma Intervention Study (AGIS), VII: the relationship between control of intraocular pressure and visual field deterioration. Am J Ophthalmol 2000;130:429-440.

6. Lama PJ. Systemic adverse effects of beta-adrenergic blockers: an evidence-based assessment. Am J Ophthalmol 2002; 134:749-760.

7. De Jong C, Solwijk T, Kuppens E, et al. Topical timolol with and without benzalkonium chloride; epithelial permeability and autofluorescence of the cornea in glaucoma. Graefes Arch Clin Exp Ophthalmol 1994;232:221-224.

8. Mietz H, Niesen U, Krielstein GK. The effect of preservatives and antiglaucomatous medication on the histopathology of the conjunctiva. Graefes Arch Clin Exp Ophthalmol 1994;232:561-565.

9. Pisella PJ, Fillacier K, Elena P, et al. Comparison of the effects of preserved and unpreserved formulations of timolol on the ocular surface of albino rabbit. Ophthalmol Res 2000;32:3-8.

10. Pisella PJ, Pouliquen P, Baudouin C. Prevalence of ocular symptoms and signs with preserved and preservative free glaucoma medication. Br J Ophthalmol 2002;86:418-423.

11. Wilson FM. Adverse external ocular effects of topical ophthalmic medications. Surv Ophthalmol 1979;24:57-88.

12. Baudouin C, Garcher C, Haouat N, et al. Expression of inflammatory membrane markers by conjunctival cells in chronically treated patients with glaucoma. Ophthalmology 1994;101:454-460.

13. Li DQ, Tseng SC. Three patterns of cytokine expression potentially involved in epithelial-fibroblast interactions of human ocular surface. J Cella Physiol 1995;163:61-79.

14. Becker J, Salla S, Dohmen U, et al. Explorative study of interleukin levels in the human cornea. Graefes Arch Clin Exp Ophthalmol 1995;233:766-771.
15. Taylor PC. Anti-TNF therapy for rheumatoid arthritis and other inflammatory diseases. Mol Biotechnol 2001;19:153-168.

16. Sfikakis PP. Behcet's disease: a new target for anti-tumor necrosis factor treatment [review]. Ann Rheum Dis 2002; 61( suppl 2):ii51-ii53.

17. Arend WP. The mode of action of cytokine inhibitors [review]. J Rheumatol Suppl 2002;65:16-21.

18. Bron AJ, Evans VE, Smith JA. Grading of corneal and conjunctival staining in the context of other dry eye tests. Cornea 2003;22:640-649.

19. Pisella PJ, Debbasch C, Hamard P, et al. Conjunctival proinflammatory and proapoptotic effects of latanoprost and preserved and unpreserved timolol: an ex vivo and in vitro study. Invest Ophthalmol Vis Sci 2004;45:1360-1367.

20. Girard MT, Matsubara M, Fini ME. Transforming growth factor- $\beta$ and interleukin-1 modulate metalloproteinase expression by corneal stromal cells. Invest Ophthalmol Vis Sci 1991;32:2441-2454.

21. Niewderkorn JY, Peeler JS, Mellon J. Phagocytosis of particulate antigens by corneal epithelial cells stimulates interleukin-1 secretion and migration of Langerhans cells into the central cornea. Reg Immunolog 1989;2:83-90.

22. Wilson SE, He YG, Weng J, et al. Epithelial injury induces keratocyte apoptosis: hypothesized role for the interleukin-1 system in the modulation of corneal tissue organization and wound healing. Exp Eye Res 1996;62:325-338.

23. Dayer JM. The pivotal role of interleukin-1 in the clinical manifestations of rheumatoid arthritis [review]. Rheumatology (Oxford) 2003;42(suppl 2):ii3-ii10.

24. Nakamura Y, Sotozono C, Kinoshita S. Inflammatory cytokines in normal human tears. Curr Eye Res 1998;17:673-676.

25. Solomon A, Dursun D, Liu Zuguo, et al. Pro- and antiinflammatory forms of interleukin-1 in the tear fluid and conjunctiva of patients with dry-eye disease. Invest Ophthalmol Vis Sci 2003;42:2283-2292.

26. Wilson WS, Duncan AJ, Jay JL. Effect of benzalkonium chloride on the stability of the precorneal film in rabbit and man. Br J Ophthalmol 1975;59:667-669.

27. Broadway DC, Chang LP. Trabeculectomy, risk factors for failure and the preoperative state of the conjunctiva [review]. J Glaucoma 2001;10:237-249.

28. Lavin MJ, Wormald RPL, Migdal CS, Hitchings RA. The influence of prior therapy on the success of trabeculectomy. Arch Ophthalmol 1990;108:1543-1548. 